\title{
Wirken Risikofaktoren beim Kind im Erwachsenenalter noch nach?
}

\begin{abstract}
Der Effekt von kindlichen Risikofaktoren auf kardiovaskuläre Erkrankungen beim Erwachsenen sowie auf Erwachsenenmortalität und -morbidität ist bisher unzureichend verstanden. Es ist bekannt, dass BMI, Glukosetoleranz, Blutdruck und Lipidstoffwechsel einflussreiche Risikofaktoren auf das kardiovaskuläre Risikoprofil sind. Der zeitliche Zusammenhang zwischen dem Auftreten des Risikofaktors und dem Auftreten eines kardiovaskulären Ereignisses wird kontrovers diskutiert.
\end{abstract}

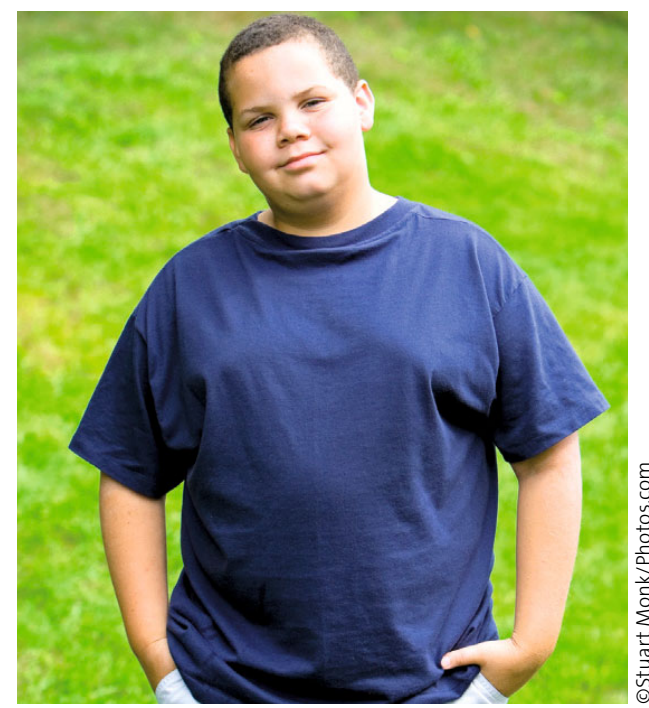

Die überzähligen Pfunde wirken sich noch im Erwachsenenalter aus.

— Die Frage, wie der Zusammenhang zwischen kindlichen Risikofaktoren und adulter Gesundheit zu sehen ist, wurde in der vorliegenden Studie untersucht. Dazu wurde eine Kohorte von 4857 indianisch-amerikanischen Kindern ohne Diabetes im mittleren Alter von 11,3 Jahren, die zwischen 1945 und 1984 geboren wurden, herangezogen. Im Schnitt erhielt jeder Studienproband drei Untersuchungen, bei denen standardisiert BMI, Glukosetoleranz, Blutdruck und Lipidspiegel analysiert wurden. Outcome war ein frühzeitiges kardiovaskuläres Ereignis vor dem 55. Lebensjahr.

3,4\% der Kohorte (166 Personen) starben an einem kardiovaskulären Ereignis nach mittlerem Follow-up von 23,9 Jahren. Kinder mit dem BMI in der höchsten Quartile hatten 2,3-mal häufiger ein kardiovaskuläres Ereignis als Kinder mit dem BMI in der untersten Quartile. Die Quartile der schlechtesten Glukoseintoleranz bewirkte eine 73\%ige Erhöhung von kardiovaskulären Ereignissen im späteren Lebensalter. Kein Zusammenhang wurde zwischen kindlichem Cholesterinspiegel und späterem kardiovaskulärem Ereignis gesehen. Kindlicher Hypertonus war allerdings direkt mit einer 57\%igen Erhöhung von frühzeitigem kardiovaskulärem Tod im Erwachsenenalter assoziiert.

\section{Kommenta}

Die Autoren fassen als Ergebnisse zusammen, dass Übergewicht, Glukoseintoleranz und Bluthochdruck bei Kindern stark und signifikant mit erhöhten Raten von frühzeitigem kardiovaskulärem Tod beim Erwachsenen assoziiert sind. Im Gegensatz dazu zeigt kindliche Hypercholesterinämie keinen Zusammenhang mit frühem Erwachsenentod. Überraschend ist, dass eine Dyslipidämie bei Kindern weniger relevant ist als eine Dysglykämie. Von allen Risikofaktoren ist allerdings Übergewicht bei Kindern der prädiktiv stärkste und relevanteste Risikofaktor. Diese Studie zeigt eigentlich, dass im Hinblick auf die Relevanz von metabolischen Risikofaktoren kaum Unterschiede zwischen Kindern und Erwachsenen bestehen.

P. SCHWARZ

- P. W. Franks et al.

Childhood obesity, other cardiovascular risk factors, and premature death. N. Engl. J. Med. 362 (2010) 485-493 